\title{
An Efficient Over-the-Cell Channel Router
}

\author{
Jong-Sheng Cherng, Sao-Jie Chen, Chieh Changfan \\ Dept. of Electrical Engineering \\ National Taiwan University \\ Taipei, Taiwan 107, ROC
}

\author{
$\mathrm{Yu}$-Hen $\mathrm{Hu}$ \\ Dept. of Electrical \& Computer Eng. \\ University of Wisconsin-Madison \\ Madison, WI 53706, USA
}

\begin{abstract}
A new approach for over-the-cell channel routing in the standard cell design siyle is proposed. This approach uses a two-layer routing model where channel is divided into zones. Each zone has a zone density and a pseudo zone density. In the proposed algorithm, the removal of any subnet from a zone will reduce the pseudo zone density by one, but only the removal of all subnets of a net from a zone will contribute to the reduction in channel density. Besides the above heuristics, techniques such as net (pin) shifting will be applied to the routing problem to further reduce channel height. Experimentally, our approach reduces more channel density than previous researches and can speed up the process of over-the-cell routing.
\end{abstract}

\section{Introduction}

Channel routing is a fundamental and very important step in the automatic layout design of VLSI circuits. For the standard cell design style, the traditional channel routing problem usually assumes that there are two layers of materials available for routing. Using this traditional routing model, many channel routers can produce solutions with quality equal to or very close to the optimal solutions for most channels. Therefore, the work of further reduction of routing area is not easy and has to rely on other new routing models, such as over-the-cell (OTC) routing [1] [2] [3].

In an over-the-cell channel router, it is assumed that one extra routing layer is available over the cells, that is, routing can be completed within the channel and over the cells. Hence, this routing model is different from that of conventional channel routing for its routing region is expanded. Therefore, an over-the-cell channel router is capable of using fewer tracks, and hence reducing total chip area. As more metal layers become available for routing in the standard cell design technology, routing over the cells becomes both practical and important.

The basic idea of the over-the-cell channel routing is to select a sel of nets for over the cells and the routing of these nets must be single layer and planar. While those nets not chosen for over the cells are routed within the channel. In this paper, we develop a new heuristic algorithm of over-the-cell channel routing for the standard cell design style. The main idea of our approach is to divide channel into zones where each zone has a zone density and a pseudo zone density. Only the removal of all subnets of a net from a zone will contribute to the reduction in the channel density. The concepts of "zone density" is the same as the one defined in [3] [4]. Since a multi-terminal net can be divided into two or more twoterminal subnets, and after this partitioning is performed on all nets of a channel instance, a different set of zone density can be derived from the same channel instance and we call this newly obtained zone density the "pseudo zone density". By using these two ideas of densities, whether a net is really helpful to reduce the channel density when it is routed over the cells can easily be determined.

This paper is organized as follows. Section 2 gives a brief review of previous works. Section 3 will explore our new heuristic algorithm in details. Experimental results and conclusion are presented in Sections 4 and 5 , respectively.

\section{Previous Works}

Different approaches have been proposed for over-thecell channel routing. The most important step for overthe-cell channel routing is to select a set of nets which are available for routing on rows over the cells. Hence, the emphasis is how to choose this set of nets. Cong and Liu assumed that the more nets being routed over the cells, the greater the reduction in channel density [2]. But blindly routing nets over the cells may not result in the reduction of channel density [3]. On the other hand, the limited number of over-the-cell tracks sometimes does not allow routing more nets over the cells.

To overcome the above disadvantages, it is essential to route over the cells only those nets whose removal will contribute to the reduction in channel density. Lin et al. solved this problem by formulating it as an integer linear programming problem [3]. One drawback of the integer linear programming is its computational inefficiency. When the problem size becomes large, the CPU time grows very rapidly. Another drawback of the method used in [3] is that when a net has three terminals with a 
terminal on one side and the other terminals on another side, this case cannot be correctly formulated using integer linear programming.

Observing that previous works did not use a right way to decompose a net into a set of subnets, we propose a more precise way for partitioning multi-terminal nets into two-terminal subnets. The novel aspects of our work are (1) pseudo zone density is used to choose the right nets for over the cells; (2) the whole channel height can be further reduced by using vacant terminals, this process is called by us "net (pin) shifting". Experimental results show that our algorithm is efficient and generates good results over previous researches. In the following section, the heuristic algorithm will be explained in details.

\section{Algorithm for OTC Channel Routing}

As mentioned in Section 1, a routing model is supposed to have two routing layers within the channel, and only a single layer over the cells for interconnections. A new algorithm for over-the-cell channel routing is developed for this two-layer model.

\subsection{Over-the-Cell Channel Routing Algorithm}

The overall algorithm is summarized in the following five major steps.

Step 1: Partition multi-terminal nets into twoterminal subnets.

This step is the preprocessing task of the whole algorithm. A good partitioning configuration can provide us with more efficient information on the choosing of a set of nets for over the cells.

Step 2: Evaluate zone density and pseudo zone density.

For any channel routing instance, the zone density of each column is first evaluated. But after the partitioning of multi-terminal nets into two-terminal subnets, the pseudo zone densities should be updated accordingly. The evaluation of pseudo zone densities is the same as that of zone densities, because we can view all the partitioned subnets as different nets and use the calculation of zone densities to find the pseudo zone densities.

Step 3: Find maximal zone density and determine the selection of nets.

Some priority rules are invented to select a set of nets which can be routed over the cells in a single layer. And we will explain in Section 3.4 that our heuristic rules are superior than previous works.

Step 4: Use net (pin) shifting to further reduce channel height.

Step 5: Generation of routing result.

The details of above steps will be explored in the following five subsections respectively.

\subsection{Partition Multi-Terminal Nets into Two-} Terminal Subnets
In a given channel routing input netlist, a net might have more than two terminals. Most routers firstly partition a multi-terminal net into two-terminal subnets and then use terminals of these subnets for routing within the channel. Our method of partitioning a multi-terminal net is different from previous researches [1] [2] [3]. Since different partitioning methods produce different sets of subnets which will have a great effect on the routing results, the step of partitioning multi-terminal nets is very important if a good routing result is required. Possible cases of partitioning multi-terminal nets into two-terminal subnets are shown in Fig. 1 and will be explained below.

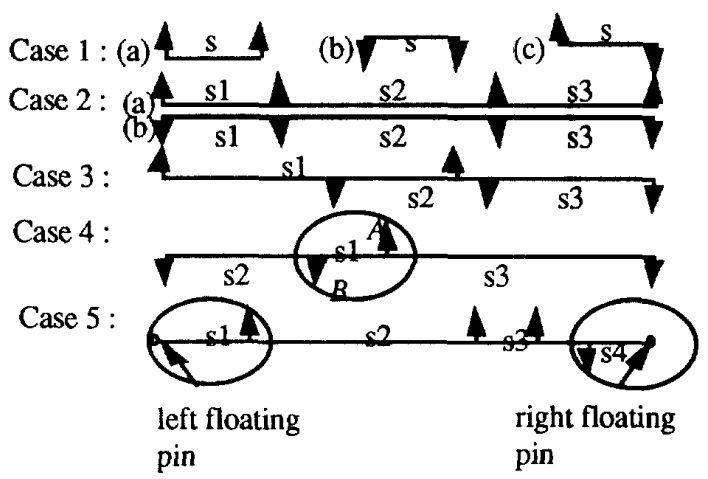

Fig. 1 The cases of partitioning multi-terminal nets into two-terminal subnets

Case 1 is trivial for two-terminal nets. Case 2 contains those multi-terminal nets with all their pins either on the top row or on the bottom row such that subnets can be partitioned as shown in Fig. 1. In Case 3, a net has more than one pin on both the top and the bottom rows, this configuration is the combination of Cases 2(a) and 2(b). Net in Case 4 has only one pin (say $A$ ) on the top row (or bottom row) and the opposite row has more than one pin. For this situation, one should choose another pin $B$ close to pin $A$ such that the length of $A$ and $B$ is horizontally minimum and these two pins can form a subnet. For channel routing, the consideration of floating pins is very important for practical applications. Case 5 will deal with this situation. The left floating pin connecting the leftmost pin forms a subnet $(s 1)$ and the right floating pin connecting the rightmost pin forms another subnet $(s 4)$. After multi-terminal nets are partitioned into proper two-terminal subnets according to the above cases, we can proceed to the Step 2.

\subsection{Evaluate Zone Density and Pseudo Zone Density}

In order to identify the critical area of a channel, a routing channel can be divided into zones of one or more column(s) according to [3]. Given a channel routing instance, we can calculate zone density for the original set 
of nets and pseudo zone density for the finally partitioned two-terminal subnets. In fact, pseudo zone density must be equal to or greater than the zone density because a multi-terminal net may produce more than one subnet. Since, the pseudo zone density for each column depends on the methods of partitioning multi-terminal nets into two-terminal subnets, once the partitioning phase in Step 1 is finished, a fixed configuration of two-terminal subnets and the values of pseudo zone density can thus be derived.

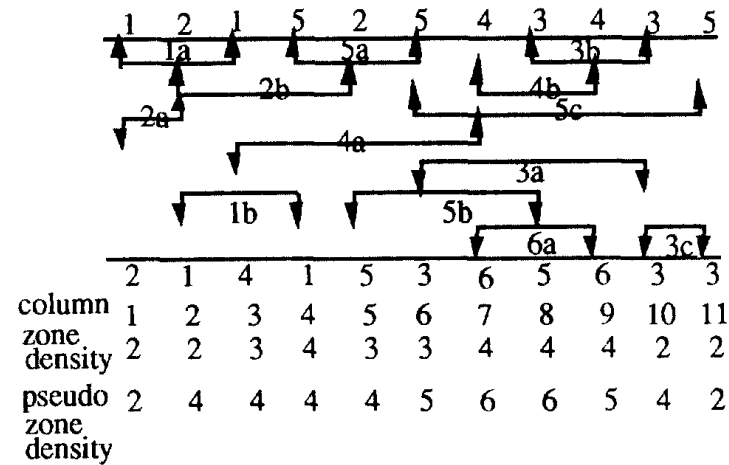

Fig. 2 The zone density and pseudo zone density of a channel

Fig. 2 depicts the results of partitioning multi-terminal nets into two-terminal subnets and shows the values of zone density and pseudo zone density in a channel. In this figure, the net intervals of net 1 , net 2 , and net 4 intersect column 3 , therefore the zone density is three for column 3 . But if viewing subnet $1 a$ and subnet $1 b$ as different nets, the pseudo zone density will increase by one. After repeating the same calculation for all other columns, pseudo zone densities can be derived for a given channel routing instance. For column 3, obviously, choosing subnet $2 b$ or subnet $4 a$ for over the cells will get more benefits than choosing subnet $1 a$ or subnet $1 b$. The reason is that unless both subnets $1 a$ and $1 b$ are routed over the cells simultaneously, otherwise if only subnet $1 a$ or $1 b$ is routed over the cells, there is no contribution to the reduction in channel density.

\subsection{Find Maximal Zone Density and Determine the Selection of Nets}

In order to reduce the density for the critical area of a channel, the zone that has maximal zone density and from which the removal of any subnet will reduce its pseudo zone density by one will be considered first. Notice that unless all subnets of a net are removed in that zone, the zone density will not be reduced. Therefore, in order to reduce the zone density in the critical area, one should carefully choose subnets to route over the cells. Six rules are derived for this purpose. Each rule has its own priority, such as Rule 1 has the highest priority, while
Rule 6 has the lowest one. The following will present these rules of processing subnet selection for routing over the cells:

Rule 1: Choose the one that has minimal number of subnets in the highest density zone.

In Fig. 2, for column 4 , net 5 has one subnet $5 a$, net 2 has one subnet $2 b$, net 4 has one subnet $4 a$ and net 1 has one subnet $1 b$; therefore, possible candidate subnet can be chosen among $5 a, 2 b, 4 a$ or $1 b$. For column 3 , net 1 has two subnets $1 a$ and $1 b$, net 2 and net 4 has one subnet $2 b$ and $4 a$ respectively. In this case, only $2 b$ or $4 a$ should be chosen for over the cells.

Rule 2: Choose the one that has minimal cross number.

In Fig. 3, observe that although net 1 has maximal length but it is crossed with nets 2, 3, 4, 5 (or nets 2,3 , 4, 5 intersect net 1). Therefore, the cross number of net 1 is equal to 4 and the cross numbers of nets $2,3,4,5$ are all equal to 1 . From this, either net 2 , net 3 , net 4 , or net 5 should be chosen for over the cells, but not net 1 .

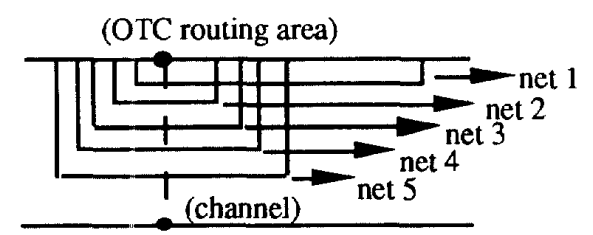

Fig. 3 The situation of intersection of five nets (The dashed line is the column with maximal zone density)

Rule 3: Choose the one that has the longest length.

To apply this rule, first, net lengths have to be calculated according to the interval width of nets. Given an interval region, the lengths of all subnets within the same region are classified into the same class, then Rule 3 will choose the net from the longest class.

Rule 4: Choose the one that can make more subnets over the cells.

In some critical zones, if there are more than one net to be chosen, choose the one that will make more subnets routed over the cells. For example, in Fig. 4, net 1, net 2 and net $3 a$ are the candidates. But since net $3 b$ can also be routed over the cells, one should choose net $3 a$ over the cells according to this rule.
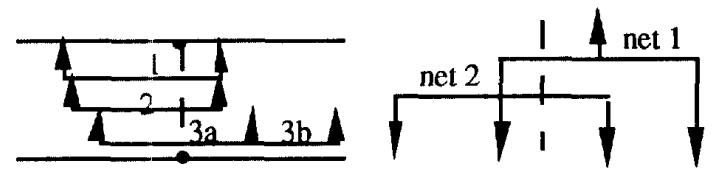

Fig. 4 Choose the one that Fig. 5 Choose two-terminal can make more subnets over nets first rather than multithe cells terminal nets 
Rule 5: Choose two-terminal nets first rather than multi-terminal nets.

After some or all subnets of a net are routed over the cells, one should have to accomplish the connectivity of a net by interconnecting a top and a bottom terminals within the channel, which is called the "connectivity preserving problem". As an example, in Fig. 5, if net 2 is chosen for over the cells, the channel density will really be reduced by one. Instead, if net 1 is routed over the cells, the channel density will not be reduced because of the connectivity preserving problem. This situation cannot be correctly handled by the integer linear programming method [3]. Fig. 6 gives an example to show this.

If the channel routing in Fig. 6 is formulated as an integer linear programming problem as in [3], one can derive nets $2,3,4,8$ or nets $2,3,4,9$ to be chosen for over the cells. But the order of choosing net 8 or net 9 over the cells is random because no information about this ordering is given in the integer linear programming formulation. First, if one chooses net 9 over the cells, one can realize the channel routing as shown in Fig. 6(a), which needs 3 tracks to complete the routing. But, if we choose net 8 over the cells, better result for this channel routing problem will be generated, which needs only 2 tracks as shown in Fig. 6(b). But when applying our Rule 5 , this situation can be handled correctly because net 8 is a two-terminal net and net 9 is a multi-terminal net.

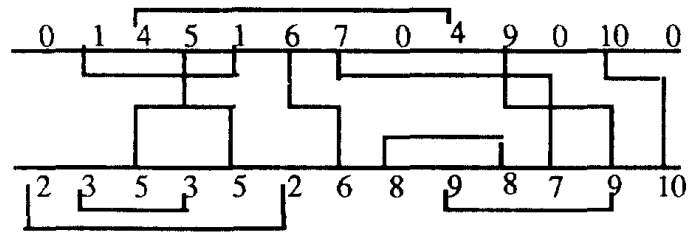

(a) net 9 over the cell

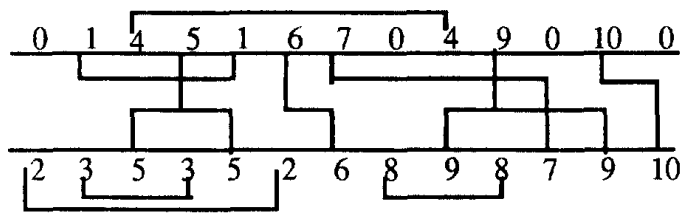

(b) net 8 over the cell

Fig. 6 A channel routing problem using Rule 5

Rule 6: The selection rules for three-terminal nets.

For a three-terminal net, its decomposition can be performed using the same rule as the Case 4 in Step 1. After decomposition, we will have two subnets, one with two terminals on the same side (subnet ss) and another with two terminals on opposite sides (subnet so). When two three-terminal nets (net 1 , net 2 ) overlap each other in some zone, choose the one (say net 1), such that its ss part can be routed over the cells, but its so subnet must not be in the interval of another net (net 2). Possible situations are shown in Fig. 7(a) to 7(e). (a)

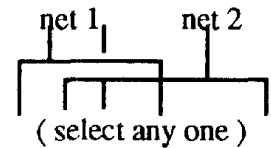

(c)

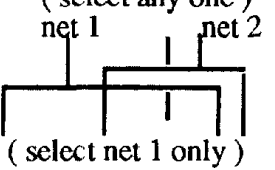

(e)

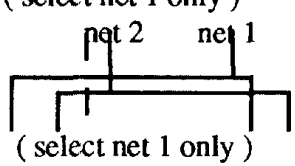

(b)

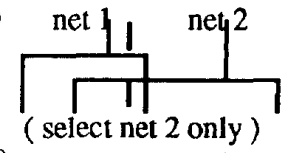

(d)

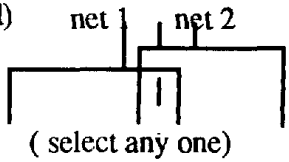

Fig. 7 The selection rules for three-terminal nets

Again, the integer linear programming formulation used in [3] cannot handle the above situations correctly. Fig. 8 gives an example to show this. First net 8 or net 9 can randomly be chosen over the cells because no information about the order of selecting net 8 or net 9 is shown in the integer linear programming formulation. If net 8 is firstly chosen over the cells, Fig. 8(a) is obtained and if net 9 is chosen, better result can be derived as shown in Fig. 8(b). Therefore, the integer linear programming formulation for these situations is ambiguous, while using our rules can solve this problem correctly and efficiently.

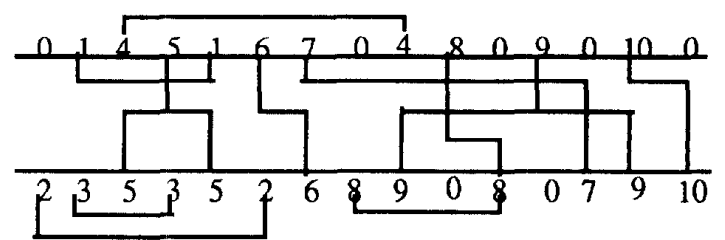

(a) net 8 over the cell

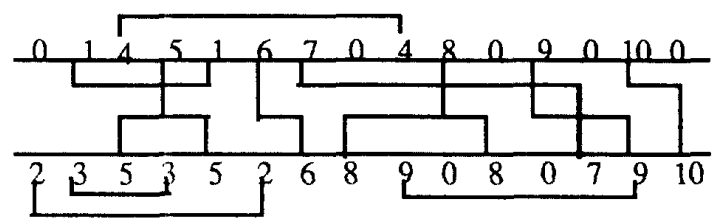

(b) net 9 over the cell

Fig. $8 \mathrm{~A}$ channel routing problem to show the usage of Rule 6

\subsection{Use Net (Pin) Shifting to Further Reduce Channel Height}

After completing the selection of nets in Step 3, net (pin) shifting through vacant terminals can be used to further reduce channel height. Knowing that most standard 
cell designs have many "vacant" terminals on the given input [5], therefore, this process will greatly help to the reduction of channel height. The processes of net and pin shiftings are depicted in Fig. 9, where Fig. 9(a) shows the case of net shifting and Fig. 9(b) the case of pin shifting. The dashed line is the original routing path and arrowed line represents the path after net (pin) shifting processing. Obviously, applying net (pin) shifting can reduce channel density.

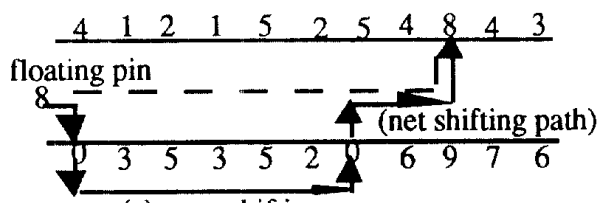

(a) a net shifting

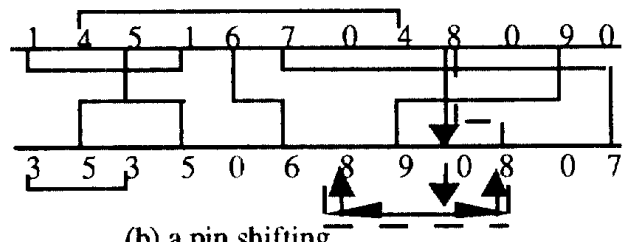

(b) a pin shifting

Fig. 9 The processes of net (pin) shifting

\subsection{Generation of Routing Result}

After performing previous steps, the original problem is now reduced to a conventional two-layer channel routing problem and an over-the-cell planar routing problem. Therefore, a planar router is developed for the routing over-the-cell and an "Independent Mode Multilayer Channel Router" [6] is used for routing within the channel. This channel router is well developed in our CAD research group and it has the capability to handle multilayer channel routing. Of course, good results and efficient running time are also provided.

\section{Experimental Results}

The over-the-cell channel router was coded in $C$ language and implemented under the Unix 4.2 BSD on a Sun4 SPARC workstation. In the following Table 1, a set of inputs from Cong [2] is tested and comparisons between our results and those of previous researches are given in the table.

Experimental results show that significant channel area reduction was achieved using our algorithm. Furthermore, the running time of each example is shorter than [3]. It seems that our over-the-cell channel router are more efficient and more practical.

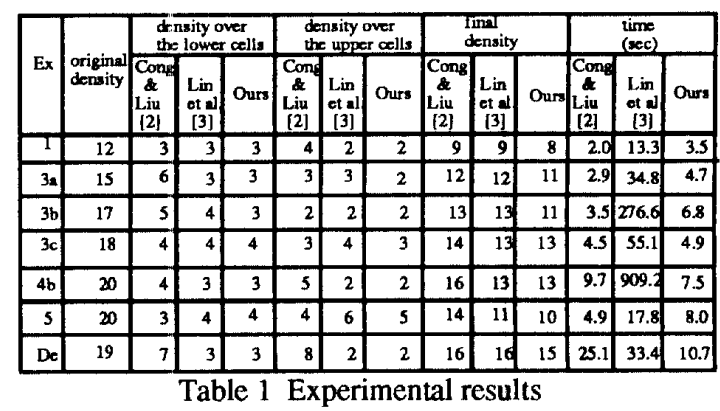

\section{Conclusions}

We have proposed a new approach for over-the-cell routing. Rules of choosing a "right" set of nets for routing over the cells were derived. Our approach utilizes the routing area over the cells more efficiently than previous researches do. Experimental results have demonstrated the superiority of our heuristic approach. The following paragraph will discuss our future works.

In the two-layer layout design, the channel routing is the most effective way to obtain a whole routing result with high density. Recently, with advances in manufacturing technology, it has been made possible to use three or more layers for interconnections (more than two metals and one polysilicon). So, it is indispensable to extend the channel router to handle multi-layered regions. Our next project is to extend the OTC channel router to multi-layer in order to further reduce channel height.

\section{References}

[1] C. L. Her, An Over-the Cell Channel Router, Master Thesis, Department of Electrical Engineering, Naticnal Taiwan University, Jun. 1991.

[2] J. S. Cong and C. L. Liu, "Over-the-Cell Channel Routing," IEEE Trans. on CAD, Vol. 9. No. 4, pp. 408-418, Apr. 1990

[3] M. S Lin, H. W. Perng, C. Y. Hwang, and Y. L. Lin, "Channel Density Reduction By Routing Over The Cell," Proc. 28th Design Automation Conference, pp. $20-125,1991$.

[4] T. Yoshimura and E. S. Kuh, "Efficient Algorithms for Channel Routing," IEEE Trans. on CAD, Vol. 1, No. L, pp. 25-35, Jan. 1982.

[5] N. D. Holmes, N. A. Sherwani, and M. Sarrafzadeh, "New algorithm for over-the-cell channel routing using vacant terminals," Proc. 28th Design Automation Conference, pp. 126-131, Jun. 1991.

[6] S. L. Lee, Enhanced Multi-layer Channel Router with Practical Considerations, Master Thesis, Department of Electrical Engineering, National Taiwan University, 1991. 